\title{
TRAYECTORIAS Y DESAFÍOS EN LA HISTORIOGRAFÍA DE LA CIENCIA Y DE LA MEDICINA EN AMÉRICA LATINA ${ }^{1}$
}

\author{
Marcos Cueto \\ Casa de Oswaldo Cruz, Fiocruz \\ Centro de Documentação e História da Saúde (CDHS) \\ Email: marcos.cueto@fiocruz.br \\ ORCID iD: https://orcid.org/0000-0002-9291-7232 \\ Matheus Alves Duarte da Silva \\ Department of Social Anthropology - University of St Andrews \\ Email: madds1@st-andrews.ac.uk \\ ORCID iD: https://orcid.org/0000-0003-0982-6231
}

Recibido: 6 noviembre 2019; Aceptado: 16 marzo 2020

Cómo citar este artículo/Citation: Cueto, Marcos; Silva, Matheus Alves Duarte da (2020) "Trayectorias y desafíos en la historiografía de la ciencia y de la medicina en América Latina", Asclepio, 72(2): p320. https://doi.org/10.3989/asclepio.2020.21

RESUMEN: El objetivo de este artículo es contribuir a la historiografía de la ciencia y la medicina Latinoamericana analizando cuatro perspectivas utilizadas por los investigadores desde mediados del siglo XX hasta comienzos del siglo XXI. La primera perspectiva fue fuertemente influenciada por un supuesto eurocéntrico según el cual la ciencia era un producto intelectual moderno, una forma de conocimiento racional superior y deseable a todas las culturas. La segunda y tercera perspectivas consideraron a la ciencia como un emprendimiento social y cultural que tenía una dinámica propia en los países latinoamericanos. Estas dos perspectivas le dieron mucha importancia a las nociones de recepción, negociación e interacción. Asimismo, resaltaron la capacidad proactiva de actores locales y nacionales. La cuarta perspectiva -asociada a una corriente más amplia conocida como Historia Global- enfatiza la circulación transnacional del conocimiento entre culturas que a veces va más allá de la región latinoamericana, desafiando algunas de las bases de las perspectivas anteriores como la centralidad del Estado Nación.

PALABRAS CLAVE: Historiografía; América Latina; Historia de la Ciencia; Historia de la Medicina; Historia Global.

TRACKS AND CHALLENGES IN THE HISTORIOGRAPHY OF THE SCIENCE AND MEDICINE IN LATIN AMERICA

ABSTRACT: The purpose of this article is to contribute to the historiography of science and medicine in Latin America by analyzing four perspectives used by researchers since the mid-20th century to the early 21st century. The first perspective was strongly influenced by an eurocentric assumption according to which science was a modern intelectual product, a higher form of rational knowledge and an aspiration of all cultures. The second and third perspectives considered science as a social and cultural venture that had a unique dynamic in Latin American countries. These two perspectives gave much importance to the notions of reception, negotiation and interaction. In addition, they highlighted the proactive capacity of local and national actors. The fourth perspective-associated to a broader perspective known as Global History-emphasizes the transnational circulation of knowledge among cultures and goes beyond the Latinamerican region, challenging some of the basis of previous perspectives such as the centrality of the Nation State.

KEY WORDS: Historiography; Latin America; History of Science; History of Medicine; Global History.

Copyright: $\odot 2020$ CSIC. Este es un artículo de acceso abierto distribuido bajo los términos de la licencia de uso y distribución Creative Commons Reconocimiento 4.0 Internacional (CC BY 4.0) 
En las últimas décadas la historia de la ciencia latinoamericana ha sido reconocida como un área de investigación legítima y creativa en las universidades de la región y entre sus pares de otros continentes. Sin embargo, sus orígenes, su diversidad y los desafíos intelectuales que enfrenta, son menos conocidos. Algunos trabajos historiográficos han querido llenar ese vacío (Armus, 2004; Azuela y Guevara Fefer, 1998; Birn y Necochea López, 2011; Edler, 2015; Espinosa, 2013; Lértora Mendoza, 1986; Saldaña, 1983; Kropf y Hochman, 2011; García, 2019; Guevara Ferfer, 2014; López Beltrán, 1997; Armus y López Denis, 2010; Fisher y Priego, 2006; Stepan, 1998). El propósito de este artículo es sumar a estos estudios analizando cuatro perspectivas que priorizaron los problemas de investigación a ser abordados. Nos concentramos a las publicaciones de Argentina, Brasil y México, así como en las investigaciones latinoamericanistas hechos por historiadores norteamericanos y europeos, por la mayor densidad de esta literatura; pero no descartamos comentar estudios relevantes producidos en otros países. Nuestro trabajo es especialmente complementario con el de Barahona, en que la autora analiza la historiografía de la biología inicialmente informada en las nociones de centro y periferia y la transición a estudios que le dan más importancia a las perspectivas transnacionales y globales (Barahona, 2018). Este artículo no es el clásico análisis historiográfico que presenta etapas cerradas y tampoco es un recuento de lo realizado en todas las áreas; algunas crecientemente especializadas como la historia ambiental (Duarte, 2013), la historia de la salud mental (Plotkin, 2001), la historia de la historia natural (Kury, 2001) y la historia de la física (Freire Junior, 2014). En nuestro análisis se intercalan la historia de la ciencia y la historia de la medicina porque muchas veces no existieron fronteras claras entre ambas. La primera - que se concentró en la historia natural- aspiraba a estudiar los conocimientos y las políticas generadas para comprender y controlar la naturaleza que cercaba a los seres humanos y la segunda -que empezó en los años cuarenta como un estudio de lo que pensaban y hacían los médicos profesionales - se fue convirtiendo en los noventa en un estudio de los saberes y las prácticas oficiales y populares sobre la salud y el cuerpo humano.

\section{LA PRIMERA PERSPECTIVA: EL AUGE DEL UNIVERSALISMO}

Aunque médicos y naturalistas del siglo XIX escribieron relatos históricos para legitimar su labor; so- lamente en los años treinta comenzaron a aparecer investigaciones cuyo objetivo principal era comprender el pasado científico. Ello ocurrió porque un grupo de profesionales - generalmente formados en ciencias o en medicina y no en historia - dedicaron la mayor parte de su tiempo al estudio, la escritura y la enseñanza en historia de la ciencia. Una profesionalización incipiente ocurrió en la Argentina que desde 1933 tenía un "Grupo de Historia de la Ciencia" que reunía a profesores universitarios como el matemático español Julio Rey Pastor (1888-1962) quien era catedrático de la Universidad de Buenos Aires (Rey Pastor, 1942). Un acontecimiento importante para ellos fue la llegada a la Argentina del historiador de la ciencia italiano Aldo Mieli (1879-1950) quien desde 1919 editaba la revista Archivio di Storia della Scienza y fue clave tanto en la organización de la Academie Internationale d'Histoire des Sciences en 1928 como en el primer congreso de historia de la ciencia realizado en París el mismo año (Chimisso 2011).

Aunque Mieli no era legalmente un refugiado, en la práctica lo era. Fue discriminado en la Europa crecientemente conservadora de los años treinta porque era judío, socialista y homosexual. Desde 1938, Mieli fue profesor en la Universidad de Santa Fé, ubicada a unas 240 millas hacia el noreste de Buenos Aires; que siguiendo la reforma universitaria argentina de 1919 promovía la enseñanza de cursos en ciencia e ingeniería. El italiano fue director de un novísimo Instituto de Historia y Filosofía de la Ciencia que contó con su espléndida biblioteca que se convirtió en una mina de oro porque en aquella época los historiadores trabajaban casi exclusivamente analizando libros. Según Mieli el Instituto Argentino podía ser el mejor del mundo; sugiriendo que podía competir con los esfuerzos del belga George Sarton quien desde la Universidad de Harvard impulsaba la Sociedad de Historia de la Ciencia e Isis (Babini, 1967). Un grupo de jóvenes lo acompañaron. Los más destacados fueron el matemático argentino José Babini (1897-1984), discípulo de Rey Pastor, quien también fue contratado en la Universidad de Santa Fé, y el emigrante húngaro Desiderio Papp (1895-1993), quien llegó a la Argentina en 1942 (Ortiz y Pyenson, 1985; Kohn, 1994). Gracias a Mieli, la revista Archeion apareció en Santa Fé entre 1940 y 1943.

Lamentablemente, desde fines de 1943 la Argentina experimentó un período de regímenes militares autoritarios con líderes antisemitas que decidieron intervenir las universidades. Mieli y Babini fueron 
despedidos sin ninguna razón explícita, pero probablemente porque el primero era judío y gay y la esposa del segundo era una líder socialista. El despido fue parte del deterioro de las universidades argentinas asaltadas por los militares. Según Kreimer, la modernización de las universidades argentinas de entonces se produjo en una sociedad marcada por inequidades y donde las élites académicas fueron víctimas de discursos populistas (Kreimer, 1996). Después de vivir en Santa Fé por menos tiempo del que esperaba, Mieli se mudó a un poblado cercano a Buenos Aires con la ayuda de un centro de exilados de la Guerra Civil Española. Desafortunadamente la continuidad de Archeion fue afectada --la revista solo reapareció en 1947 en Europa-- y los problemas de salud de Mieli se deterioraron hasta su muerte en 1950 (Asúa, 1997).

A pesar de estos problemas, Mieli, Babini y Papp se embarcaron en el ambicioso proyecto enciclopédico Panorama general de historia de la ciencia. Esta fue la primera obra general de historia de la ciencia publicada en español. El primer volumen de 1945 trataba de los "griegos y romanos" y anunciaba el eje organizador de la colección: la emergencia y desarrollo del conocimiento racional surgido en Europa Occidental (Panorama no estudiaría la ciencia hindú o china porque los autores consideraban que no tuvo trascendencia). Asimismo, lo local - o mejor dicho lo latinoamericano- no era muy importante. Babini llegó a publicar trabajos sobre el período precolombino y la historia de la ciencia en la Argentina (para diferenciarla de una ciencia argentina) alimentando estereotipos sobre los obstáculos anticientíficos: la superstición indígena, el legado escolástico de la religión católica y la precariedad del Estado. Para ellos, inmigrantes europeos del siglo XIX fueron los primeros en hacer trabajo científico verdadero (Mieli, 1952, 4-5 Babini, 1967). En un momento en que la Revolución Científica de Inglaterra del siglo XVII no era aún consagrada como un momento fundacional, el período ejemplar para Mieli era el Renacimiento y el arquetipo del conocimiento holístico, Leonardo de la Vinci (Mieli, 1950). Dos volúmenes más aparecieron antes de la muerte de Mieli en 1950. Durante los siguientes años y a partir de borradores de Mieli; Papp y Babini completaron el Panorama en diez volúmenes. Babini aprovechó su situación de no trabajar en la Universidad -adonde la enseñanza y administración podrían consumir su tiempo-- para escribir artículos periodísticos y libros en un país que experimentaba un auge editorial. Hacia el final de esa década se produjo un breve interregno democrático y en 1958 Babini fue presidente de la editorial de la Universidad de Buenos Aires (Eudeba) desde donde promovió la historia de la ciencia. Además, tradujo The History of Science and the New Humanism; un texto de Sarton, que -como Mieli aspiraba - quería reforzar la historia de la ciencia como un puente entre las humanidades y las ciencias (Sarton, 1948).

Sería fácil descalificar el trabajo de Mieli y de Babini como positivista (es decir como una interpretación teleológica del devenir histórico como el triunfo de la racionalidad). Era algo más que esto. Mieli, Babini y Papp pensaban que tenían la misión de mostrar la importancia del diálogo entre las disciplinas académicas en cualquier parte del mundo. Mieli tuvo asidua correspondencia con historiadores de la ciencia europeos y norteamericanos, y Babini fue miembro de la Academia Internacional de Historia de la Ciencia. Su trabajo tuvo además un componente misionero en la Argentina. Su convicción que existía un saber universal era como una herramienta civilizatoria y un antídoto a los estridentes discursos nacionalistas en la Argentina. Ellos creían que era un imperativo presentar un ideal al cual los argentinos podían aspirar.

Investigadores contemporáneos de otros países realizaron un trabajo similar al de Argentina y algunos tuvieron una actitud ambivalente, y hasta positiva, hacia las herencias precolombinas y coloniales. El sociólogo brasileño Fernando de Azevedo (1894-1974), profesor y fundador de la moderna Universidad de São Paulo creada en 1934, suscribía la idea de la importancia de los lazos entre las ciencias y las letras. Los dos volúmenes de su libro As Ciências no Brasil de 1956 sistematizaba el desarrollo de las disciplinas científicas establecidas (Azevedo, 1955). Una idea parecía a la de Babini de registrar el pálido camino de los saberes científicos en el devenir histórico nacional -aunque con una mayor reivindicación de los conocimientos indígenas precolombinos y en una clave marxista-la seguiría el ingeniero y filósofo de la ciencia mexicano Eli de Gortari quien publicó un libro con un título revelador: La ciencia en la Historia de México (Gortari, 1963). En el Perú de los cuarenta, profesores de la Facultad de Medicina de la Universidad de San Marcos fundaron una Sociedad de Historia de la Medicina y tuvieron nutrida correspondencia con Henry Sigerist, el suizo-norteamericano quien desde la Universidad de Johns Hopkins revolucionó la historia de la medicina (y que mantuvo correspondencia con los historiadores de la medicina de México y la Argentina 
como Izquierdo y Beltrán que ha sido analizada en otros artículos (Castañeda López y Rodríguez de Romo, 2007; Asúa, 2005). Entre los sanmarquinos estuvieron Juan B. Lastres quien publicó tres tomos de una historia de la medicina peruana cuyo primer volumen estuvo dedicado a la medicina precolombina.

Los historiadores de la medicina peruanos y mexicanos se enfrentaron a un asunto soslayado por Mieli: el legado del conocimiento indígena precolombino (seguramente porque ese legado era evidente en, por ejemplo, la trepanación craneana en los Andes). Lastres dio una solución que pareció concertar los intereses de quienes creían en la superioridad de la ciencia y la medicina europeas y quienes defendían el pasado indígena. Según el peruano, practicantes especializados de las élites de los Incas y de otras culturas precolombinas habían alcanzado un alto grado de conocimientos médicos, astronómicos y agrícolas. Lamentablemente la conquista española del siglo XVI, no solo por la violencia que implicó sino por las enfermedades nuevas que trajo a las Américas, eliminó a estos practicantes y a estas elites, y sus avanzados conocimientos se perdieron o se convirtieron en un pálido reflejo de su antiguo esplendor en los saberes populares. Entonces, según Lastres, fueron los médicos y sabios europeos que llegaron al Virreinato peruano - adonde se fundó tempranamente la Universidad de San Marcos a mediados del siglo XVI-quienes mantuvieron la continuidad del progreso del conocimiento. De esta manera, la solución de Lastres cumplía tres objetivos. Primero creaba una ilusión de progreso linear. Segundo, explicaba la relación de los saberes precolombinos con la irrelevancia de los saberes indígenas populares. En tercer lugar, enlazaba su trabajo con el tema de los "grandes médicos" que predominaba entre los historiadores norteamericanos y europeos. De hecho, Sigerist - autor de The great doctors; a biographical history of medicine una obra publicada en español en 1949- escribió a Lastres felicitándolo por su fascinante primer tomo de la "medicina Inca" y asegurando que iba a citarlo varias veces en la obra general de historia de la medicina que estaba escribiendo².

Otro ejemplo de la primera perspectiva fue José López Sánchez (1911-2004), profesor de historia de la medicina en la Universidad de La Habana y autor de biografías como la de Carlos J. Finlay. En México, el biólogo profesor de la Universidad Nacional Autónoma de México Enrique Beltrán (1903-1994) explicó en su Medio Siglo de la Ciencia Mexicana 1900-1950 de 1952 que no obstante las "viejas raíces" de la cultura mexicana, no había existido hasta años recientes "un clima favorable al progreso científico." (Beltrán, 1952, p. 13). Pero también es importante mencionar que en algunos textos Beltrán defendería los saberes precolombinos y que tuvo un papel fundamental en la organización del primer Coloquio Mexicano de Historia de la Ciencia realizado en 1963.

Todos ellos de una u otra manera comulgaron con el supuesto hegemónico -no enunciado claramente en una política consistente por parte del Departamento de Estado de los Estados Unidos-de la Fundación Rockefeller (que reorganizó la enseñanza científica y médica en buena parte de las universidades y sistemas de salud de las Américas en los años treinta y cuarenta). Según este supuesto los países científicamente "menos avanzados" tenían que imitar a los países más desarrollados en un desarrollo que era considerado lineal y progresivo adonde la racionalidad iría haciendo desaparecer la "magia" y la "superstición"; o mejor dicho en términos modernos saberes alternativos de la naturaleza y el cuerpo humano (Cueto, 1994).

Estas ideas cambiarían con otra generación de historiadores.

\section{SEGUNDA PERSPECTIVA: RECEPCIÓN Y DINÁMICAS LOCALES}

Un giro hacia lo local caracterizó la historiografía latinoamericana en los años setenta y noventa. Los conceptos clave fueron la recepción, la recreación y la adaptación locales, así como el estudio de las dinámicas institucionales locales. Según las nuevas investigaciones los latinoamericanos no fueron receptores pasivos de la ciencia europea y los factores contingentes locales, antes ignorados, eran decisivos. Esta perspectiva estuvo enmarcada por una crítica al artículo "The Spread of Western Science" de George Basalla que describía -y prescribía de una manera más explícita a como lo había hecho la Rockefeller en los años treinta y cuarenta-como la difusión cultural realizada desde sociedades industrializadas era decisiva para la modernización científica de los países en desarrollo (Basalla, 1967). Según Basalla, muchos de estos países estaban en una etapa de "ciencia colonial" enfrascada en la imitación de la ciencia de los centros metropolitanos europeos y los Estados Uni- 
dos. Su modelo reflejaba las políticas norteamericanas de la Guerra Fría que buscaban la hegemonía cultural en el hemisferio occidental y la supervisión del desarrollo (Rostow, 1960). Se esperaba que la influencia norteamericana fuese un agente de cambio en la medicina y la ciencia latinoamericanas, de una manera parecida al papel que se creía habían tenido la investigación alemana en las universidades norteamericanas del siglo XIX.

Historiadores latinoamericanos alineados con esta segunda perspectiva criticaron la interpretación de Basalla como eurocéntrica y lineal. Varios asistieron al Congreso Internacional de Historia de la Ciencia que se realizó en Bucarest (1981) y se comprometieron a realizar actividades en la región. En agosto del año siguiente varios de ellos se reunieron en Puebla, México, para organizar la Sociedad Latinoamericana de Historia de la Ciencia y Tecnología (SLHCT). En 1982, la Sociedad empezó a publicar en Ciudad de México la revista Quipu, teniendo como editor a Juan José Saldaña; un discípulo de Rene Taton, que estuvo en Bucarest y había sido nombrado Presidente de la SLHCT (un cargo en el que se mantendría por varios años). Quipu sería una referencia hasta fines de la década de 1990 (Silva, 2014). Al referirse a una técnica precolombina de llevar cuentas, el título sugería que el desarrollo científico regional tenia raíces nativas (Saldaña, 1984). Nuevas o renovadas sociedades nacionales de historia de la ciencia -las más importantes fueron las de México, Cuba, Colombia, Venezuela y Brasil -alentaron reuniones con regularidad como el Seminario Internacional para el Estudio de la Metodología de la Historia Social de las Ciencias en América Latina realizado en noviembre de 1983 en Bogotá. De una manera algo independiente a la SLHCT, Elías Trabulse, quien era químico, doctor en Historia y profesor en el prestigioso Colegio de México, venía produciendo valiosos estudios sobre la dinámica local de la ciencia colonial mexicana y de su desarrollo en el siglo XIX (Trabulse, 1984).

La investigación, los lectores y el contexto de estos estudios sobre el desarrollo de la ciencia nacional tuvieron elementos en común. Desde comienzos de los años sesenta intelectuales y científicos renombrados de América Latina criticaron los programas de transferencia de ciencia y tecnología que suponían un desarrollo imitativo de los países desarrollados. Algunos estaban interesados en elaborar políticas coherentes para los relativamente recientes consejos nacionales de investigación o creían que una ciencia nacionalmen- te comprometida con el desarrollo promovería una industrialización menos dependiente del exterior. El supuesto de muchos trabajos era que una historia políticamente orientada de la ciencia contribuiría al desarrollo. Muchos historiadores estuvieron influenciados por la Teoría de la Dependencia que argumentaba que los latinoamericanos podían escapar de un diseño imperial que dictaba que el flujo de materias primas de la "periferia" (o países "subdesarrollados") estaba destinado a enriquecer el "centro" (o los países industrializados) (Prebisch 1981). Historiadores de la ciencia de América Latina empezaron a prestar atención a los esfuerzos por superar la tendencia a utilizar la región como un enclave científico adonde los europeos recogían información que sería procesada en Europa y publicada en francés, alemán o inglés.

Varios historiadores redescubrieron el libro La disputa del nuevo mundo: historia de una polémica, 1750-1900 de Antonello Gerbi (publicado en italiano en 1955 y en español en 1960) quien mostraba que los latinoamericanos polemizaron con los naturalistas europeos que pintaban a la fauna y flora de las Américas como inferior a los modelos ideales del viejo continente (Gerbi, 1960). La segunda perspectiva también implicó un cambio metodológico al utilizar archivos, fuentes documentales primarias y entrevistas orales. Los historiadores latinoamericanos se alimentaron del giro social y constructivista que atravesaba la historia de la ciencia en distintas partes del mundo y comenzaron a entender la ciencia como un proceso de construcción de discursos, organizaciones y prácticas, más que un simple producto. Esta fue la preocupación del sociólogo Schwartzman en su recuento del desarrollo científico brasileño desde las postrimerías del período colonial que buscaba valorar y promover un contexto político favorable para las instituciones de investigación (publicado en portugués en 1977 y reeditado en el 2001; Schwartzman, 2001). Esta segunda perspectiva se consolidó en el Brasil con los profesores Motoyama y Dantes del Departamento de Historia de la Universidad de São Paulo (Ferri y Motoyama, 1979). Dantes y sus alumnos estudiaron no solo universidades, también museos, laboratorios privados, jardines botánicos y centros de investigación, lo cual era novedoso en relación al trabajo de Azevedo e importante en el Brasil, país adonde la creación de las universidades recién ocurrió en el siglo XX (Dantes, 2001).

Otra característica de esta perspectiva fueron las alianzas con investigadores de Estados Unidos y Eu- 
ropa. Entre los norteamericanos interesados en la historia latinoamericana estuvieron la profesora de la Universidad de Columbia Nancy Stepan (posteriormente Nancy Leys Stepan) y Thomas F. Glick, primero profesor de la Universidad de Texas y después de la de Boston. Coincidió con ellos uno de los pioneros en el análisis del tema de ciencia e imperialismo: el historiador norteamericano Lewis Pyenson, entonces profesor en la Universidad de Montreal. A ellos se sumó Nathan Reingold del Instituto Smithsonian-que, si bien no trabajó en archivos latinoamericanos como Stepan, Pyenson o Glick, publicó sobre ciencia y colonialismo y tuvo contacto regular con los latinoamericanos. Al mismo tiempo, los investigadores de la región participaron en mayor número de eventos internacionales como los congresos de historia de la ciencia de Berkeley en 1985, de Hamburgo en 1989 y de Zaragoza en 1993.

Un modelo de la segunda perspectiva fue el libro de Stepan sobre el instituto que fundó en Río de Janeiro el microbiólogo Oswaldo Cruz a comienzos del siglo XX. Según Stepan, esta fue una institución médica de primer nivel porque adaptó creativamente el modelo francés pasteuriano (Cruz estudió en el Instituto Pasteur en París). Los científicos locales consiguieron legitimar la bacteriología en el Estado como parte de los programas sanitarios de control de enfermedades y de discursos sobre el desarrollo del país. Su libro no fue una ruptura radical con las ideas de Basalla sobre la ciencia colonial. Stepan consideraba que el brasileño era un caso inusual de la "ciencia colonial" que había predominado durante el siglo XIX (por eso el uso de "Beginnings" en el título). Pero según ella, la transición de la ciencia colonial a la ciencia nacional era compleja y se debía sobre todo a factores locales (Stepan, 1976). Otros estudios siguieron a Stepan y demostraron que la institucionalización científica se entrelazaba con discursos de la utilidad de la investigación en la educación, el comercio, el crecimiento poblacional o el desarrollo económico. Estas investigaciones enfatizaron la idea que no toda la ciencia de la periferia era periférica al conocimiento local y mundial (Cueto, 1989; Peard, 1999).

Pyenson vinculó la recepción de las ciencias exactas, especialmente la física y la astronomía en Buenos Aires y Córdoba, con el imperialismo alemán, holandés y francés de fines del siglo XIX. Su interpretación privilegiaba al imperialismo más que a la recepción local; según Pyenson las motivaciones imperialistas obstaculizaron la apropiación local (Pyenson, 1985,
1989, 1993). Glick, quien ya tenía una trayectoria en la historia de la tecnología medieval española, editó un libro seminal sobre la recepción del Darwinismo, que incluyó un capítulo sobre México escrito por Roberto Moreno de los Arcos (Glick, 1988; Domingues, Sá, Glick, 2003). Es importante mencionar que Moreno de los Arcos fue un distinguido historiador mexicano cuyo trabajo -como otros lo harían despuésfue un puente entre la primera y la segunda perspectiva mencionada en este artículo (Azuela, 1997). Posteriormente, Glick analizaría la recepción de otros paradigmas, como la Teoría de la Relatividad y el Psicoanálisis y esbozó un marco teórico sobre la recepción en el que los factores locales eran fundamentales (Glick, 1987). No menos importante fue que animó a otros estudios de recepción (Domingues, Sá y Glick 2003). Los latinoamericanos también establecieron lazos con investigadores españoles como José Luis Peset, Antonio LaFuente, Horacio Capel, José Sala Catalá y Leoncio López Ocón, quienes estudiaban las expediciones científicas, los legados de la ciencia borbónica del siglo XVIII y la relación entre ciencia y nacionalismo (Lafuente y Mazuecos, 1987; Lafuente, Elena, y Ortega, 1993). Gracias a esta relación se realizó la primera reunión de Historia de la Ciencia y de la Técnica de los Países Ibéricos e Iberoamericanos en Madrid en septiembre de 1984 con participación de varios historiadores de la región (Peset, 1985). Asimismo, ellos mantuvieron contacto con investigadores franceses como Patrick Petitjean, Anne Marie Moulin y Catherine Jami, quienes organizaron en 1990 en París el coloquio "Ciencia e Imperios" que comparaba países asiáticos, africanos, americanos y de Oceanía (Petitjean, Jami, Moulin, 1992). El tema del evento se convirtió en una importante materia de investigación durante los siguientes años. Al mismo tiempo, Roy McLeod y David Wade Chambers de Australia tuvieron un importante rol al participar en reuniones en América Latina, cuestionar el modelo difusionista de Basalla y presentar versiones complejas del imperialismo científico y de la ciencia nacional (Chambers, 1987). En parte por estas alianzas con investigadores de otros países se empezó a dar mayor importancia a nuevos archivos en Europa y en Estados Unidos. El Rockefeller Archive Center de Nueva York, que conservaba los papeles de la Fundación Rockefeller activa en la región desde 1918, se convirtió en un repositorio central para analizar la norteamericanización de la ciencia y la medicina latinoamericanas (Cueto, 1994; Birn, 2006). 
Otra característica de esta segunda perspectiva fue enfocarse en pocas instituciones. Ello tuvo ventajas y riesgos. Fue productivo porque en la mayoría de los países latinoamericanos los discursos y las prácticas científicas se concentraron en unas cuantas ciudades e instituciones adonde la creatividad científica se manifestó no solo en las ideas sino en la capacidad de los investigadores de enfrentarse a condiciones adversas muchas veces improvisando instrumentos $y$ encontrando estabilidad en su trabajo. El riesgo de escribir historias locales fue que estas acabaron siendo a veces parroquiales y no siempre estaba clara la relevancia internacional de los científicos latinoamericanos. Por otro lado, el énfasis en la recepción local no objetaba las grandes narrativas que suponían que los paradigmas científicos se habían generado en el exterior. Otra desventaja fue que a pesar de que se trató de reconstruir las conexiones regionales; las investigaciones comparativas acabaron siendo historias paralelas similares con puntos de conexión no claramente establecidos.

De todas maneras, en esta segunda perspectiva el número de historiadores de la ciencia latinoamericanos creció y modernizó --muchos de ellos a diferencia de la primera perspectiva eran formados en historia y ya no en las ciencias. Un reflejo de lo anterior fueron el segundo y tercer congreso de historia de la ciencia y la tecnología latinoamericanas, realizados en La Habana en 1985 y São Paulo en 1988 por la SLHCT. Finalmente, el legado de esta segunda perspectiva se sintió hasta el 2001 en el Congreso Internacional de Historia de la Ciencia que se realizó en México con el provocador título: "Ciencia y Diversidad Cultural" (De Greiff y Nieto, 2008).

\section{LA TERCERA PERSPECTIVA: REDES INTERNACIONALES Y NUEVOS ACTORES SOCIALES}

La tercera perspectiva se concentró en dos grandes temas. Primero, la interacción de científicos de un país específico de Latinoamérica con sus pares europeos o norteamericanos; poniendo énfasis en la capacidad de los primeros en ser protagonistas de redes internacionales. En segundo lugar, la importancia de actores no profesionales -como periodistas, curanderos y charlatanes-en discusiones que trascendían los ámbitos universitarios. La inspiración teórica provino delos innovadores estudios de actor-redes de Bruno Latour y Michel Callon (Latour, 1984; 2003; Callon,
2006). Aunque los trabajos de esta perspectiva se multiplicaron con mayor intensidad durante la década de los noventa, el contexto que los explican se remonta a mediados de la década de 1980 cuando comenzó el derrumbe de los regímenes militares de la región. Movimientos sociales civiles en los que participaban sindicatos, Organizaciones No Gubernamentales, y grupos feministas trabajaban en un mundo globalizado adonde las distancias parecían disminuir al ritmo acelerado de las tecnologías de la informática y de la comunicación. Nuevas preocupaciones, como el medio ambiente en que cada vez había más estudiosos, influenciaron la historia de la ciencia. Ninguna localidad parecía remota y una diversidad de voces y actores parecían indispensables para sustentar la democracia que regresaba a la región. Fue también importante que, en temas como el SIDA y el medio ambiente intervinieran no solamente expertos sino activistas que desafiaban a las autoridades. En parte por este contexto, los historiadores de la región prestaron atención a los saberes populares sobre el cuerpo, la enfermedad y la naturaleza (Chalhoub, 1996; Sampaio, 2001). Los debates científicos atrajeron la atención porque eran una lupa que magnificaba disputas que trascendían los marcos profesionales convencionales y revelaban la injerencia del género, la raza y la pobreza en el quehacer científico. También se estudió cómo las historias locales se entrelazaban con fuerzas metropolitanas e imperiales y cuyos protagonistas participaban activamente en redes del conocimiento. Esta tercera perspectiva disputó la validez de nociones binarias de ciencia moderna vs ciencia colonial y cuestionó el supuesto que factores contingentes locales explicasen por sí solos dinámicas científicas nacionales (López Beltrán, 1997). Asimismo, a veces, los estudios comparativos adquirieron entonces cierta relevancia (Stepan, 1991).

Esta perspectiva coincidió con un desarrollo más consistente de programas académicos, eventos y publicaciones especializadas. En 1994 se creó en Río de Janeiro la publicación periódica História, Ciências, Saúde-Manguinhos y la revista Saber y Tiempo fue lanzada en Buenos Aires dos años después. Una nueva generación de historiadores estuvo influenciada por estudios feministas, subalternos y postmodernos con cuyos referentes no siempre tenían un contacto regular (como Bruno Latour, Pierre Bourdieu, Gian Prakash y Mary Louise Pratt). Hubo también un mayor diálogo entre la historia de las ciencia y marcos teóricos de la historia social, como la micro historia ita- 
liana y la historia de las clases populares "desde abajo" de E. P. Thompson. Algunos investigadores europeos, como llana Löwy, cultivaron una relación cercana con América Latina. (Löwy, 1990; 2001). Entonces, se consolidaron subáreas de investigación como la historia de la medicina, la historia de la ciencia natural y la historia de las ciencias exactas. Sobre estas últimas las disciplinas que recibieron más atención fueron la historia de la física, la historia de la geografía y la historia de la geología (Figueirôa, 1997). Poco a poco la interacción de la historia de la ciencia con la historia ambiental se convirtió en una preocupación central de los investigadores. Un problema de la profesionalización de la historia de la ciencia latinoamericana y su reconocimiento en las universidades vino con los problemas generados por una hiperespecialización; fragmentación y una multiplicación de debates, a veces complejos y limitados. Ello en parte explica porque, después de un congreso de historia de la ciencia y la tecnología realizado en Río de Janeiro de 1998, la SLHCT se fue diluyendo hasta extinguirse, y los historiadores expertos en subtemas comenzaron a reunirse en reuniones especializadas.

De cualquier manera, esta perspectiva produjo libros importantes. Un ejemplo fue el de Benchimol sobre la compleja transición de las ideas miasmáticas a las bacteriológicas en Brasil. Benchimol se concentraba en un "perdedor" del pasteurianismo, Domingos José Freire, quien ganó reconocimiento en redes transnacionales pero que sería posteriormente suplantado en la historia canónica de la microbiología nacional por Oswaldo Cruz. Benchimol describió las estrategias heterodoxas de Freire para legitimar sus ideas (como sus artículos periodísticos, sus publicaciones en francés en la capital carioca y sus presentaciones en teatros) revelando la frágil frontera entre los saberes académicos y populares (Benchimol, 1999). Otro ejemplo de esta perspectiva fue el libro sobre las epidemias del Perú durante el siglo XX por Cueto, que ganó el Premio Iberoamericano del Latin American Studies Association, que confrontó las prácticas y discursos de diferentes actores ante las principales epidemias (como los médicos, el Estado, los religiosos, y las clases populares) (Cueto, 1997). De una manera parecida, Armus analizó las ansiedades populares en la búsqueda de medicamentos contra la tuberculosis en la Argentina y Agostoni, la popularidad de los sanadores religiosos mexicanos a comienzos del siglo XX (Armus, 2007; Agostoni, 2018). Vinculados a lo anterior estuvieron las investigaciones sobre las repuestas a las endemias y epidemias (Ca- rrillo, 2005), el surgimiento de políticas oficiales sanitarias y la profesionalización en la medicina-en especial de áreas poco estudiadas hasta entonces como la enfermería lo que permitió incorporar un enfoque de género (Ramacciotti, 2009; Hochman, 2012; Biernat et al., 2015). La intención de vincular estrechamente lo local y lo global apareció en el libro de Rodríguez sobre la criminología en la Argentina. Ella analizó el trabajo del inmigrante croata Juan Vucetich, quien diseñó un sistema de identificación mediante impresiones digitales, que fue adoptado por la policía y sanitaristas de otras partes del mundo y que moduló la definición de ciudadanía en la Argentina (Rodríguez, 2006). Un libro que avizoraban la nueva-cuarta-- perspectiva de las investigaciones fue el de Nieto sobre la botánica, la farmacia y la medicina de fines del período colonial en Colombia que le daba atención tanto al contexto comercial y político español como al de las colonias americanas para explicar la disputa del poder económico, social e intelectual (Nieto Olarte, 2000). Asimismo, este tipo de análisis a los dos lados del Atlántico fue cultivado con brillo por Cañizares-Esguerra, autor de estudios sobre el período científico colonial (CañizaresEsguerra, 2001).

\section{LOS DESAFÍOS DE UNA CUARTA PERSPECTIVA: EL GIRO GLOBAL}

En los últimos años un giro global ha impactado en la historia en general y en la historia de la ciencia. (Conrad, 2016; Barahona, 2018; Subrahmanyam, 1997; Gruzinski, 2004; Raj, 2010; Conrad, 2016; Suárez Diaz y Mateos, 2016). Esta perspectiva se propone examinar el movimiento transnacional de documentos, libros, mapas, materiales de laboratorio, artefactos técnicos, imágenes e ideas de diferentes países, entre distintas culturas e inclusive en diferentes idiomas (Safier, 2010). El objetivo es trascender las fronteras nacionales y no limitarse a lo que hacía -o dejaba de hacer- el Estado Nación. Complementariamente, esta perspectiva se concentra en los personajes intermediarios, como comerciantes, misioneros, divulgadores y viajeros, que hicieron inteligibles productos científicos en diferentes culturas (Schaffer, 2009). Un supuesto importante es que el contenido del conocimiento se transforma en la circulación del mismo (Raj, 2013). Un comentario pertinente es que los autores difieren entre enfatizar la fluidez de la circulación de conocimientos o en resaltar los obstáculos 
que esta circulación encuentra y la necesidad de registrar las adaptaciones del mismo (Secord, 2004).

El contexto de estos estudios es todavía la era de la globalización que también influyó en la perspectiva anterior. Sin embargo, la cuarta perspectiva apareció durante la intensificación de procesos políticos y económicos algo diferentes como la franca debilidad de los Estados en los países en desarrollo, el poder de las corporaciones transnacionales y la visibilidad de las corrientes antiglobalización. Nuevos estudios históricos intentan ser supranacionales y demostrar cómo la generación y reconfiguración del conocimiento no están constreñidos a marcos nacionales. Como bien señala Cañizares-Esguerra lo "global" y lo "local" no son nociones excluyentes, sino que presuponían una a la otra (Cañizares-Esguerra, 2006). De una manera parecida otros estudios buscan escapar a la dualidad "centro" y "periferia" e identificar los itinerarios, conexiones, encuentros y desencuentros culturales y políticos. $Y$ también algunos estudios tratan de trascender el ámbito latinoamericano para colocar en el centro de su análisis localidades asiáticas o africanas cuestionando el supuesto que la región tenga características culturales únicas. Paradójicamente con esta perspectiva han aparecido estudios comprensivos que abarcan toda la historia de la ciencia o de la medicina de un país, de la región o de una disciplina pero que a diferencia de los panoramas generales producidos anteriormente tratan de enfocarse en un problema de investigación como el nacionalismo, la raza o las limitaciones políticas de la salud pública (Amador, 2015; Hochman y Di Liscia, 2012; Carbonetti, Garcés, y Blanco, 2002; Agostoni y Speckman Guerra, 2005; Zárate, 2008; Quevedo, 2008; Cueto y Palmer, 2015).

Algunos trabajos ejemplifican esta perspectiva. Al analizar los programas médicos de la Rockefeller en varios países de Centroamérica y el Caribe, Palmer reescribió la historia de la agencia filantrópica y prestó atención a los cambios en su agenda imperialista por presiones de actores locales postcoloniales no profesionales como los curanderos. El estudio de Palmer indica que la producción y el consumo del conocimiento fue local y global simultáneamente (Palmer, 2010). Otro ejemplo de esta simultaneidad es el libro de Soto Laveaga que tiene un título provocador "Los laboratorios de la Selva." Ella analiza como en el México de los cuarenta, cuando se descubrió que el tubérculo conocido como barbasco era indispensable para la producción del primer anticonceptivo oral, los campesinos, que conocían bien esta planta, negociaron con científicos, farmacéuticas multinacionales y con el gobierno mexicano para hacer valer sus derechos (Soto Laveaga, 2009). Ella regresa a un tema mal tratado por los argentinos y peruanos de mediados del siglo XX: la importancia fundamental de los conocimientos populares en la formación de los saberes y las políticas oficiales y en la globalización de conocimientos (Gómez, 2017). Este libro también coincide con un tema registrado años atrás por el investigador ecuatoriano Eduardo Estrella. En su estudio de las expediciones científicas en los Andes sobre el poder curativo de la quina, Estrella encontró que los europeos invisibilizaron en sus publicaciones oficiales el papel de guías y curanderos nativos. De esta manera, aunque el trabajo científico había implicado ideas y prácticas indígenas; estas acabaron escondidas. (Estrella, 1995). El trabajo de Rosenblat sobre México subraya los intercambios transnacionales para entender la construcción de categorías raciales y políticas de asimilación de los indígenas a comienzos del siglo XX (Rosemblatt, 2018). Además, otros temas han sido estudiados en una perspectiva transnacional como la eugenesia, los públicos de la ciencia y la movilidad del conocimiento (Miranda y Vallejo, 2012; Correa, Kottow, Vetö, 2016; Sanhueza Cerda, 2017). De una manera parecida, pero para el periodo colonial, Gómez estudio las ideas y practicas sobre el cuerpo, el clima y la naturaleza entre esclavos y exesclavos del Caribe quienes eran consultados por jesuitas, farmacéuticos, médicos y curanderos indígenas de distintos orígenes. Su libro - que recibió el William $\mathrm{H}$. Welch Medal de la Asociación de Historia de la Medicina en Estados Unidos - es además una crítica a la noción tradicional de la "Revolución Científica" como un fenómeno exclusivamente europeo que ignora el conocimiento generado en prácticas populares durante el siglo XVII. En el caso del Caribe estudiado por Gómez, estas prácticas priorizaban la experiencia empírica sobre el dogma, privilegiaban el eclecticismo, y consideraban al olfato y los otros sentidos fundamentales en la legitimación del conocimiento (Gómez, 2017). Recientemente, Matheus Duarte da Silva mostró que el surgimiento de dos laboratorios brasileños de comienzos del siglo XX (Manguinhos en Rio de Janeiro y Butantan en São Paulo) fue parte de una circulación de saberes sobre la peste bubónica en la que participaron activamente el Instituto Pasteur de Paris y laboratorios en Bombay, entonces una colonia británica. Duarte da Silva estudió la dinámica e interacción transnacional entre centros ubicados en Brasil, Fran- 
cia y la India alrededor de la producción de conocimiento sobre la peste. Duarte da Silva cuestiona algunos supuestos sobre la emergencia casi autosuficiente de la microbiología brasileña, mostrando la importancia de la India e investigadores franceses en los conocimientos elaborados en el Brasil (Silva, 2018; 2020).

Sin embargo, esta cuarta perspectiva tiene problemas pendientes que aparecieron en perspectivas anteriores como el eclecticismo y la falta de consistencia. Esto último significa que la glorificación de la Historia Global es muchas veces retórica. En parte porque la definición de esta Historia no está clara como tampoco están claros los términos de historia transnacional del conocimiento o histoire croisée usados en algunas investigaciones-- algunos estudios anuncian en su introducción que van a hacer una historia global, pero después repiten perspectivas anteriores como la recepción de paradigmas internacionales. Otro asunto irresuelto es la relación entre la Historia Global de la Salud y la Historia de la Salud Global (esta última, que es más común, puede ser un estudio del período posterior a la Guerra Fría y la primera una historia transnacional de la salud). Asimismo, nuevos paradigmas sanitarios que quieren reemplazar la Salud Global, como la Salud Planetaria apoyada con entusiasmo por la revista Lancet y la Fundación Rockefeller desde 2015-todavía no parecen haber reclutado a historiadores latinoamericanos a diferencia de otras regiones del así llamado "Sur Global" (Dunk et al., 2019). Por otro lado, no están claras las ventajas para los latinoamericanos de deshacerse de una dimensión regional. Es cierto que América Latina fue un concepto creado por Francia en el siglo XIX; pero también es verdad que durante el siglo XX el populismo, el autoritarismo y las inequidades sociales hicieron a los países latinoamericanos más parecidos. Asimismo, por lo menos desde la década del ochenta los frecuentes contactos entre historiadores de la región han creado una densidad de conocimiento que no debería ser desperdiciada. Coincidimos con otros autores que defienden la idea de la relevancia de fronteras culturales regionales en el estudio histórico de la circulación mundial del conocimiento (Fan, 2007).

Otro problema para instaurar esta perspectiva es que persiste una fuerte tradición para trabajar en archivos nacionales y dialogar con historiadores especializados en ellos. Una razón que explica lo anterior es que los historiadores latinoamericanos tienen problemas en obtener recursos financieros para visitar archivos metropolitanos que se encuentran en países desarrollados; esenciales para una visión transnacional (una dificultad que se incrementa porque algunos consulados exigen costosos trámites y tortuosos formularios para obtener una visa). Otra razón es el idioma. Buena parte de los historiadores latinoamericanos no tienen la fluidez necesaria como para investigar documentos en el exterior en un idioma diferente al suyo (Paz, 2016). Es último está vinculado a su visibilidad internacional porque casi no publican en el latín de la ciencia moderna; el inglés. Finalmente, otro problema por el cual no se consolida el giro global es por el difícil contexto político actual. En la secuela de la crisis económica del 2008, el populismo de derecha ha criticado la globalización, minado los recursos para las universidades y alentado los ataques irracionales a la ciencia; incluyendo a las humanidades y la historia.

\section{CONCLUSIÓN}

En la primera perspectiva analizada en este artículo existe un esfuerzo por hacer de la historia una herramienta que coloque a América Latina a la par de procesos intelectuales y profesionales europeos y norteamericanos. La segunda y tercera perspectivas no objetan por completo una jerarquía adonde la ciencia y la medicina de los Estados Unidos y Europa sean el ápice. Sin embargo, enfatizan la búsqueda del conocimiento como una empresa social y cultural que tiene una dinámica propia en los países latinoamericanos y resaltan la capacidad de los actores locales de adaptar y transformar influencias foráneas. Estas dos perspectivas han tenido y siguen teniendo una gran influencia en la investigación. Aunque la cuarta perspectiva coincida en la atención a las dimensiones culturales y sociales; no las considera separadas de la historia intelectual de la ciencia y desafía algunas de las bases intelectuales de las perspectivas anteriores. Por ejemplo, no asume una primacía del viejo continente ni una subalternariedad de América Latina como hechos consumados, le interesa las urdimbres internacionales más que las dinámicas nacionales, le da atención a los intermediarios transnacionales y busca revelar procesos invisibilizados en las historiografías tradicionales. Asimismo, supone que la construcción de jerarquías y de un liderazgo científico global, anclado en las metrópolis de los países industrializados, es un proceso global en la que intervinie- 
ron los científicos y otros actores de América Latina.

Estas perspectivas presentan visiones a veces contrastantes, pero no necesariamente antagónicas. De hecho, las cuatro coexisten hasta el día de hoy. Un aspecto en común de las tres últimas es el desafío de explicar la relación entre lo local y lo global (como se reflejó en el subtítulo "Between the Global and the Local" del congreso internacional de historia de la ciencia realizado en Río de Janeiro en 2017). Es

\section{NOTAS}

1 Una versión preliminar de este trabajo fue leida por Marcos Cueto en la ceremonia de inauguración del 250 Congreso Internacional de Historia de la Ciencia y la Tecnología (ICHST) realizado en Río de Janeiro el 23 de julio del 2017.

\section{BIBLIOGRAFÍA}

Agostoni, Claudia; Speckman Guerra, Elisa (eds.) (2005), De normas y transgresiones: enfermedad y crimen en América Latina, 1850-1950, México, D.F., Universidad Nacional Autónoma de México.

Agostoni, Claudia, (2018), “Ofertas médicas, curanderos y la opinión pública: el Niño Fidencio en el México posrevolucionario", Anuario Colombiano de Historia Social y de la Cultura, 45(1), pp.215-243.

Amador, José (2015), Medicine and nation building in the Americas, 1890-1940, Nashville, Vanderbilt University Press.

Armus, Diego (2004), "Legados y tendencias en la historiografía sobre la enfermedad en América Latina moderna". En: Márquez Valderrama, Jorge; Casas Orrego, Álvaro; Estrada Orrego, Victoria Eugenia (eds.), Higienizar, medicar, gobernar: historia, medicina y sociedad en Colombia, Medellín, Universidad Nacional de Colombia, pp.13-39.

Armus, Diego (2007), La ciudad impura: salud, tuberculosis y cultura en Buenos Aires, 1870-1950, Buenos Aires, Edhasa.

Armus, Diego; López Denis, Adrián (2010), “Disease, medicine and health". En: Moya, José (ed.), The Oxford Handbook of Latin American History, New York, Oxford University Press, pp. 424-453.

Asúa, Miguel de (1997), "Morir en Buenos Aires. Los últimos años de Aldo Mieli", Saber y tiempo, 1 (3), pp. 275-292.

Asúa, Miguel de (2005), "Henry Sigerist and the History of Medicine in Latin America: his Correspondence with Juan R. Beltrán", Bulletin of the History of Medicine, 79, pp. 111-117.

Azevedo, Fernando de (1955), As ciências no Brasil, Rio de Janeiro, Melhoramentos. probable que en el futuro más historiadores latinoamericanos se aventuren en la cuarta perspectiva o se dediquen a la atractiva historia del medio ambiente. $Y$ también que investigadores tengan una actitud ecléctica que combine algunas perspectivas. En todo caso, esperamos que al estudiar la historia de la historia de la ciencia como pretende este artículo, puedan conocer mejor los problemas, las posibilidades y los desafíos que existieron y que existen.

2 Henry Sigerist a Juan B Lastres, 13 de Agosto de 1948, MS 799, Henry Sigerist papers, Box 16. Folder "Lastres, Juan B", Manuscripts and Archives, Yale University Library, New Haven, Estados Unidos.

Azuela, Luz Fernanda (1997), "Roberto Moreno de los Arcos y la historia de las ciencias", Ciencia Ergo Sum, 4 (1), pp. 119-120.

Azuela, Luz Fernanda; Guevara Fefer, Rafael (1998), "La ciencia en México en el siglo XIX: una aproximación historiográfica", Asclepio, 50 (2), pp. 77-105.

Babini, José (1962), “Aldo Mieli y la historia de la ciencia en la Argentina", Physis, 4, pp. 64-84.

Babini, José (1967), La prehistoria de la ciencia, Buenos Aires, Centro Editor de América Latina.

Barahona, Ana (2018), "Local, Global, and Transnational Perspectives on the History of Biology," en Dietrich, Michael R.; Borrello, Mark \& Solomon Harman, Oren (eds), Handbook of the Historiography of Biology, Cham, Springer.

Basalla, George (1967), "The spread of western science”, Science, 156 (3775), pp. 611-622.

Beltrán, Enrique (1952), Medio siglo de ciencia mexicana, 19001950, México, Secretaría de Educación Pública.

Benchimol, Jaime L. (1999), Dos micróbios aos mosquitos: febre amarela e a revolução pasteuriana no Brasil, Rio de Janeiro, Fiocruz.

Biernat, Carolina; Cerdá, Juan Manuel; Ramacciotti, Karina Inés y Juan Manuel Cerdá, (eds.) (2015), La salud pública y la enfermería en la Argentina, Bernal, Universidad Nacional de Quilmes.

Birn, Anne-Emanuelle (2006), Marriage of convenience: Rockefeller international health and revolutionary Mexico, Rochester, University of Rochester Press.

Birn, Anne-Emanuelle; Necochea López, Raúl (2011), “Footprints on the future: looking forward to the history of health 
and medicine in Latin America in the twenty-first century", Hispanic American Historical Review, 91 (3), pp. 503-527.

Callon, Michel (2006), "Sociologie de l'acteur réseau". En: Akrich, Madeleine; Callon, Michel; Latour, Bruno (eds.), Sociologie de la traduction: textes fondateurs, Paris: École des mines de Paris, pp. 267-276.

Cañizares-Esguerra, Jorge (2001), How to write the history of the New World: histories, epistemologies, and identities in the eighteenth-century Atlantic world, Stanford, Stanford University Press.

Cañizares-Esguerra, Jorge (2006), Nature, empire, and nation: explorations of the history of science in the Iberian world, Stanford, Stanford University Press.

Carbonetti, Adrián; Garcés, Carlos Alberto; Blanco, Fernando (ed.) (2002), De sujetos, definiciones y fronteras: ensayos sobre disciplinamiento, marginación y exclusión en América, de la colonia al siglo XX, Córdoba, Ferreyra.

Carrillo, Ana Maria, (2005), “ ¿Estado de peste o estado de sitio?: Sinaloa y Baja California, 1902-1903", Historia Mexicana, 54(4), pp.1049-1103.

Castañeda López, Gabriela y Rodríguez de Romo, Ana Cecilia (2007), "Henry Sigerist y José Joaquín Izquierdo: dos actitudes frente a la historia de la medicina en el siglo XX", Historia Mexicana, 57, 1, pp.139-191.

Chalhoub, Sidney (1996), Cidade febril: cortiços e epidemias na corte imperial, São Paulo, Companhia das Letras.

Chambers, David Wade (1987), 'Period and process in colonial and national science'. En: Reingold, Nathan; Rothenberg, Marc (eds.), Scientific colonialism: a cross-cultural comparison, Washington, D.C., Smithsonian Institution Press, pp. 297-321.

Chimisso, Cristina (2011), "Fleeing dictatorship: socialism, sexuality and the history of science in the life of Aldo Mieli", History Workshop Journal, 72 (1), pp. 31-51.

Conrad, Sebastian (2016), What is global history?, Princeton, Princeton University Press.

Correa, María José; Kottow, Andrea; Silvana Vetö, Silvana (eds.) (2016), Ciencia y espectáculo: circulación de saberes científicos en América Latina, siglos XIX y XX, Santiago de Chile, Ocho Libros Editores.

Cueto, Marcos (1989), Excelencia científica en la periferia: actividades científicas e investigación biomédica en el Perú, 1890-1950, Lima, GRADE.

Cueto, Marcos, (ed.) (1994), Missionaries of science: the Rockefeller Foundation and Latin America, Bloomington, Indiana University Press.

Cueto, Marcos (1997), El regreso de las epidemias: salud y sociedad en el Perú del siglo XX, Lima, Instituto de Estudios Peruanos.

Cueto, Marcos; Palmer, Steven Paul (2015), Medicine and public health in Latin America: a history, New York, Cambridge University Press.

Dantes, Maria Amélia M., (org.) (2001), Espaços da ciência no Brasil: 1800-1930, Rio de Janeiro, Fiocruz.
De Greiff A., Alexis; Nieto, Mauricio (2008), "Lo que aún no sabemos sobre el intercambio tecnocientifico entre Sur y Norte: nortecentrismo, difusión científica y estudios sociales de la ciencia". En: Gorbach, Frida; López Beltrán, Carlos, Saberes locales: ensayos sobre historia de la ciencia en América Latina, Zamora, El Colegio de Michoacán, pp. 41-70.

Domingues, Heloisa Maria Bertol; Sá, Magali Romero; Glick, Thomas F. (eds.) (2003), A recepção do darwinismo no Brasil, Rio de Janeiro, Fiocruz.

Duarte, Regina Horta. 2013. "Between the national and the universal: natural history networks in Latin America in the nineteenth and twentieth centuries", Isis, 104 (4), pp. 777-787.

Dunk, James H.; Jones, David S.; Capon, Anthony; Anderson, Warwick H. (2019), "Human Health on an Ailing Planet Historical Perspectives on Our Future", New England Journal of Medicine, 381, pp. 778-782.

Edler, Flavio Coelho, (2015), "A história das ciências e seus públicos", Revista Maracanan, (13), pp. 23-33.

Espinosa, Mariola (2013), "Globalizing the history of disease, medicine and public health in Latin America", Isis, 104 (4), pp. $798-806$.

Estrella, Eduardo (1995), “Ciencia ilustrada y saber popular en el conocimiento de la quina en el siglo XVIII". En: Cueto, Marcos (ed.), Saberes andinos: ciencia y tecnología en Bolivia, Ecuador y Perú, Lima, Instituto de Estudios Peruanos, pp. 37-58.

Fan, Fa-ti (2007), "Science in cultural borderlands: methodological reflections on the study of science, European imperialism, and cultural encounter", East Asian Science, Technology and Society, 1 (2), pp. 213-231.

Ferri, Mário Guimarães; Motoyama, Shozo (coord.) (1979), História das ciências no Brasil, São Paulo, Editora da Universidade de São Paulo.

Figueirôa, Silvia Fernanda de Mendonça (1997), As ciências geológicas no Brasil: uma história social e institucional, 1875-1934, São Paulo, Hucitec.

Fisher, John; Priego, Natalia (2006), “Ignorance and 'habitus': blinkered and enlightened approaches towards the history of science in Latin America", Bulletin of Latin American Research, 25 (4), pp. 528-540.

Freire Junior, Olival (2014), The quantum dissidents: rebuilding the foundations of quantum mechanics (1950-1990), Berlin, Springer.

García, Mónica (2019), "La historiografía de la fiebre amarilla en América Latina desde 1980: los límites del presentismo", História, Ciências, Saúde-Manguinhos, 26 (2), pp. 623-641.

Gerbi, Antonello (1960), La disputa del nuevo mundo: historia de una polémica, 1750-1900, México: Fondo de Cultura Económica.

Glick, Thomas F. (1987), "La transferencia de las revoluciones científicas a través de las fronteras culturales", Ciencia y Desarrollo, 12 (72), pp. 77-89. 
Glick, Thomas F. (ed.) (1988), The comparative reception of Darwinism, Chicago: University of Chicago Press.

Gómez, Pablo F. (2017), The experiential Caribbean: creating knowledge and healing in the early modern Atlantic, Chapel Hill, University of North Carolina Press.

Gortari, Eli de. (1963), La ciencia en la historia de México, México: Fondo de Cultura Económica.

Gruzinski, Serge (2004), Les quatre parties du monde: histoire d'une mondialisation, Paris, Martinière.

Guevara Ferfer, Rafael. (2014), El Uso de la Historia en el Quehacer Científico, Mexico, Universidad Nacional Autonoma de Mexico.

Hochman, Gilberto (2012), A era do saneamento: as bases da política de saúde pública no Brasil. São Paulo: Hucitec.

Hochman, Gilberto; Di Liscia, María Silvia; Palmer, Steven Paul (eds.) (2012), Patologías de la patria: enfermedades, enfermos y nación en América Latina, Buenos Aires, Lugar Editorial.

Kohn Loncarica, Alfredo (1994). “Eloge: Desiderio Papp, 21 May 1895-31 January 1993", Isis, 85 (4), pp-666-667.

Kreimer, Pablo (1996), "Science and politics in Latin America: the old and the new context in Argentina", Science, Technology and Society, 1 (2), pp. 267-289.

Kropf, Simone; Hochman, Gilberto (2011), "From the beginnings: debates on the history of science in Brazil", Hispanic American Historical Review, 91 (3), pp. 391-408.

Kury, Lorelai Brilhante (2001), Histoire naturelle et voyages scientifiques 1780-1830, Paris, L'Harmattan.

Lafuente, Antonio; Elena, Alberto; Ortega, María Luisa (eds.) (1993), Mundialización de la ciencia y cultura nacional: actas del Congreso Internacional "Ciencia, Descubrimiento y Mundo Colonial", Madrid, Doce Calles.

Lafuente, Antonio; Mazuecos, Antonio (1987), Los caballeros del punto fijo: ciencia, política y aventura en la expedición geodésica hispanofrancesa al virreinato del Perú en el siglo XVIII, Barcelona, Ediciones del Serbal.

Latour, Bruno (1984), Les microbes: guerre et paix, Paris: A. M. Métailié.

Latour, Bruno (2003), Science in action: how to follow scientists and engineers through society, Cambridge, Harvard University Press.

Lértora Mendoza, Celina Ana (1986), "Los estudios de historia de la ciencia en Argentina", Quipu, 3 (1), pp. 135-147.

López Beltrán, Carlos (1997), “Ciencia en los márgenes: una reconsideración de la asimetría centro-periferia". En: Rutsch, Mechthild; Serrano, Carlos (ed.), Ciencia en los márgenes: ensayos de historia de las ciencias en México, México, D.F., Universidad Nacional Autónoma de México, Instituto de Investigaciones Antropológicas, pp. 19-31.

Löwy, Ilana (1990), "Yellow fever in Rio de Janeiro and the Pasteur Institute Mission (1901-1905): The transfer of science to the periphery", Medical History, 34 (2), pp 144-163.
Löwy, Ilana (2001), Virus, moustiques et modernité: la fièvre jaune au Brésil, entre science et politique, Paris, Editions des archives contemporaines.

Mateos, Gisela \& Suárez-Díaz, Edna, (eds.) (2016), Aproximaciones a lo local y lo global: América Latina en la historia de la ciencia, México: Centro de Estudios Filosóficos, Políticos y Sociales Vicente Lombardo Toledano.

Mieli, Aldo (1950), Panorama general de historia de la ciencia. Volumen 4. Lionardo da Vinci, sabio, Buenos Aires: Espasa-Calpe.

Mieli, Aldo (1952), Panorama general de historia de la ciencia. Volumen 1. El Mundo Antiguo: griegos y romanos, Buenos Aires, Espasa-Calpe.

Miranda, Marisa; Vallejo, Gustavo (eds.) (2012), Una historia de la eugenesia: Argentina y las redes biopolíticas internacionales, 1912-1945, Buenos Aires, Editorial Biblos.

Nieto Olarte, Mauricio (2000), Remedios para el imperio: historia natural y la apropiación del Nuevo Mundo, Bogotá, Instituto Colombiano de Antropología e Historia.

Ortiz, Eduardo L.; Pyenson, Lewis (1985), “Eloge: José Babini, 11 May 1897-18 May 1984", Isis, 76 (4), pp. 567-569.

Palmer, Steven Paul (2010), Launching global health: the Caribbean odyssey of the Rockefeller Foundation, Ann Arbor, University of Michigan Press.

Paz, Gustavo L. (2016), "Global history and Latin American history: a comment", Almanack, (14), pp. 118-124.

Peard, Julyan G. (1999), Race, place, and medicine: the idea of the tropics in nineteenth century Brazilian medicine, Durham, Duke University Press.

Peset Reig, José Luis (ed.) (1985) La ciencia moderna y el Nuevo Mundo: actas de la I Reunión de Historia de la Ciencia y de la Técnica de los Países Ibéricos e Iberoamericanos (Madrid, 25 a 28 de septiembre de 1984). Madrid, Consejo Superior de Investigaciones Científicas y Sociedad Latinoamericana de Historia de las Ciencias y de la Tecnología.

Petitjean, Patrick; Jami, Catherine; Moulin, Anne Marie (eds.) (1992), Science and empires: historical studies about scientific development and European expansion, Dordrecht, Springer Science.

Plotkin, Mariano Ben (2001), Freud in the Pampas: the emergence and development of a psychoanalytic culture in Argentina, Stanford, Stanford University Press.

Prebisch, Raúl (1981), "The Latin American periphery in the global system of capitalism", Cepal Review, (13), pp. 143-150.

Pyenson, Lewis (1985), Cultural imperialism and exact sciences: German expansion overseas, 1900-1930, New York, Peter Lang.

Pyenson, Lewis (1989), Empire of reason: exact sciences in Indonesia, 1840-1940, Leiden, E.J. Brill.

Pyenson, Lewis (1993), Civilizing mission: exact sciences and French overseas expansion, 1830-1940, Baltimore, Johns Hopkins University Press. 
Quevedo, Emilio (et. al.) (2008), Historia de la medicina en Colombia. Tomo II, Bogotá, Tecnoquímicas.

Raj, Kapil (2010), Relocating modern science: circulation and the construction of knowledge in South Asia and Europe, 1650-1900, Basingstoke, Palgrave Macmillan.

Raj, Kapil (2013), "Beyond Postcolonialism... and Postpositivism: circulation and the global history of science", Isis, 104 (2), pp. 337-347.

Ramacciotti, Karina Inés (2009), La política sanitaria del peronismo, Buenos Aires, Editorial Biblos.

Rey Pastor, Julio (1942), La ciencia y la técnica en el descubrimiento de América, Buenos Aires, Espasa-Calpe.

Rodríguez, Julia (2006), Civilizing Argentina: science, medicine, and the modern state, Chapel Hill, University of North Carolina Press.

Rosemblatt, Karin Alejandra (2018), The science and politics of race in Mexico and the United States, 1910-1950, Chapel Hill, University of North Carolina Press.

Rostow, Walt Whitman (1960), The stages of economic growth: a non-Communist manifesto, Cambridge, Cambridge University Press.

Safier, Neil (2010), "Global knowledge on the move: itineraries, Amerindian narratives, and deep histories of science", Isis, 101 (1), pp. 133-145.

Sanhueza Cerda, Carlos (2017), La movilidad del saber científico en América Latina: objetos, prácticas e instituciones (siglos XVIII al XX), Santiago de Chile, Editorial Universitaria.

Saldaña, Juan José (1983), “Hacia una crítica histórica, teórica y metodológica de la historiografía latinoamericana de las ciencias", Ciencia Tecnología y Desarrollo, 7 (3), pp. 333-346.

Saldaña, Juan José (1984), “Presentación”, Quipu, 1 (1), pp. 5-6.

Sampaio, Gabriela dos Reis (2001), Nas trincheiras da cura: as diferentes medicinas no Rio de Janeiro imperial, Campinas, Editora Unicamp.

Sarton, George (1948), Historia de la ciencia y nuevo humanismo, Rosario, Editorial Rosario.
Schaffer, Simon; Roberts, Lissa; Raj, Kapil; Delbourgo, James (eds.) (2009), The brokered world: go-betweens and global intelligence, 1770-1820, Sagamore Beach, MA, Science History Publications.

Schwartzman, Simon (2001), Um espaço para a ciência: a formação da comunidade científica no Brasil, Brasília, Ministério de Ciência e Tecnologia.

Secord, James, (2004), "Knowledge in transit" Isis 95 (4), pp. 654-672.

Silva, Márcia Regina Barros da (2014), "História e historiografia das ciências latino-americanas: Quipu (1984-2000), Revista Brasileira de História da Ciência, 7 (1), pp. 47-57.

Silva, Matheus Alves Duarte da (2018), "De Bombaim ao Rio de Janeiro: circulação do conhecimento e a criação do Laboratório de Manguinhos, 1894-1902", História, Ciências, Saúde-Manguinhos 25 (3), pp. 639-657.

Silva, Matheus Alves Duarte da (2020), Quand la peste connectait le monde : production et circulation de savoirs microbiologiques entre Brésil, Inde et France (1894-1922), Thèse (Ph.d.) - École des Hautes Études en Sciences Sociales, Paris. 2020.

Soto Laveaga, Gabriela (2009), Jungle laboratories: Mexican peasants, national projects, and the making of the Pill, Durham, Duke University Press.

Stepan, Nancy (1976), Beginnings of Brazilian science: Oswaldo Cruz, medical research and policy, 1890-1920, New York, Science History Publications.

Stepan, Nancy (1991), "The hour of eugenics": race, gender and nation in Latin America, Ithaca, Cornell University Press.

Stepan, Nancy (1998), "Tropical medicine and public health in Latin America”, Medical History, 42 (1), pp. 104-112.

Subrahmanyam, Sanjay (1997), "Connected histories: notes towards a reconfiguration of early modern Eurasia", Modern Asian Studies, 31 (3), pp. 735-762.

Trabulse, Elías (1984), El círculo roto, México, D.F., Fondo de Cultura Económica.

Zárate C., María Soledad (comp.) (2008), Por la salud del cuerpo: historia y políticas sanitarias en Chile, Santiago de Chile, Universidad Alberto Hurtado. 